\title{
An RFID-based tracking system gives mice their say
}

Habedank, A., Urmersbach, B., Kahnau, P., and Lewejohann, L. Behav Res Methods https://doi.org/10.3758/s13428-021-01593-7 (2021)

Your average laboratory mouse will spend most of its time in the confines of its home cage, so why not make that comfortable for them? "The best that we can do for refinement, in my view, is to refine housing conditions," says Lars Lewejohann, an animal welfare professor at the German Center for the Protection of Laboratory Animals (Bf3R) who has focused much of his career on that third 'R.' To figure out what's best, Lewejohann's lab likes to ask the mice what they prefer. To help automate such preference tests - in which mice are given different husbandry and/or enrichment options and then monitored for their preference - the lab has developed the Mouse Position Surveillance System (MoPSS).

Existing options to track mice can be costly, says lead author Anne Habedank - commercial tracking systems can be expensive to purchase, while analyzing video recordings can eat up hours. There are tools to help automate video analyses, but these can still struggle with identifying individuals in a group if the animals become obscured
- a problem, Habedank notes, when testing enrichment items such as nesting material and running wheels. For the MoPSS, they combine radio frequency identification (RFID) equipment with an inexpensive Arduino microcontroller that notes whenever a mouse - identity confirmed by a small subdermal transponder - passes by an RFID reader.

To test mouse preferences, the lab connected standard cages with a simple plastic tube that contained an RFID reader at each end to record when a mouse entered and left the different sides of the set-up, which will contain different forms of enrichment. Mice however are fast - often too fast for the readers, in fact, says Habedank. To slow them down long enough for the readers to successfully log their movement, the team restricted the ends of the tunnel with small barriers; additionally, they wrote an R script that could identify where a mouse was last 'seen,' should it make it past undetected.

In their proof-of-principle publication, the team used the MoPSS to test the bedding preference of 12 group-housed female C57BL/6J mice over the span of a week; a months-long experiment to test mouse preference for different active enrichment devices is currently underway. As the system records continuously, it may also help researchers \& animal staff track activity patterns and circadian rhythms, says Lewejohann - if an abnormal pattern is detected, the animal could be given a closer look for potential health issues.

All in, the MoPSS cost the lab less than 150 Euros to build, and they provide detailed instructions and parameters for constructing and running it in Behavioral Research Methods. They hope it will be an easy, inexpensive option to help other labs give their mice their say.

Ellen P. Neff

Published online: 8 September 2021

https://doi.org/10.1038/s41684-021-00855-3

\section{Virus-Antibody-Free Guinea Pigs}

"Serving the research community for over 50 years. Guinea Pig Production is Our Business...

- Excellent quality

- Outstanding service Our Only Business"

- Genuine concern for each customer's particular needs
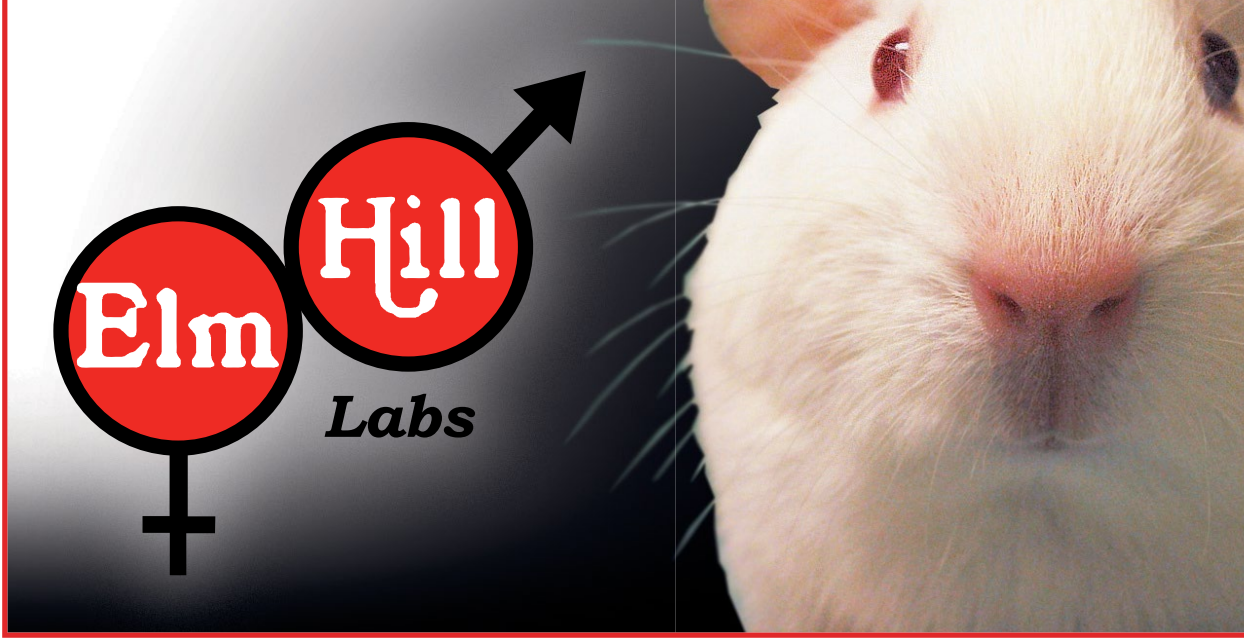

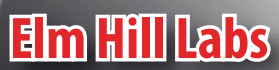
7 Kidder Road Chelmsford, MA 01824

Phone. (800) 941-4349

Fax. (978) 256-2545

Email.ehlabs@comcast.net

Web. www.elmhilllabs.com

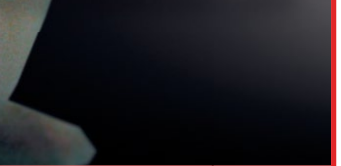

